\title{
ANÁLISE DA PRODUÇÃO DE EMBRIÕES NA FERTILIZAÇÃO IN VITRO E TRANSFERÊNCIA DE EMBRIÕES PARA DOADORAS NELORE
}

\author{
Renato Travassos Beltrame, ${ }^{1}$ Celia Raquel Quirino, ${ }^{2}$ Luis Gustavo Barioni, ${ }^{3}$ \\ Ângelo José Burla Dias ${ }^{4}$ e Paulo Marcelo de Souza ${ }^{5}$ \\ 1. Doutorando em Ciência Animal, LRMGA, CCTA, UENF. E-mail: rtbeltrame@terra.com.br \\ 2. Professor associado da Universidade Estadual do Norte Fluminense \\ 3. Pesquisador doutor da Embrapa Cerrados \\ 4. Professor associado da Universidade Estadual do Norte Fluminense \\ 5. Professor associado da Universidade Estadual do Norte Fluminense.
}

\section{RESUMO}

Ajustou-se uma função de densidade probabilidade para o número de embriões viáveis produzidos após fertilização in vitro em doadoras da raça Nelore, a partir de dados fornecidos pela Associação Brasileira de Criadores de Zebu $(\mathrm{ABCZ})$, referente à análise de 20.619 doadoras, 71.602 aspirações e um total de 509.643 embriões. Modelou-se a densidade probabilidade do número de embriões viáveis mediante a função exponencial, executando-se a determinação dos parâmetros por meio da máxima verossimilhança, em um método de gradiente não linear. O nível de precisão obtido foi de $\mathrm{RMSE}=0,040$ e $\mathrm{R}^{2}=0,98$, para a representação da probabilidade do número de embriões viáveis produzidos por doadoras Nelore na técnica de fertilização in vitro(FIV). Para comparar os modelos (curvas de probabilidade de transferência de embriões ajustada por Beltrame, em 2006, e de FIV, neste trabalho), aplicou-se a técnica de comparação de curvas com o teste F (Silva e Azevedo, 2002). Não foram encontradas diferenças entre as curvas do número de embriões viáveis obtidos após coleta e produzidos após aspiração de doadoras na raça Nelore. Ainda, sugere-se a existência de um fator único limitante que afete biologicamente a produção de embriões nas técnicas de transferência de embriões e fertilização in vitro.

PALAVRAS-CHAVES: Banco de dados, densidade probabilidade, doadoras, simulação.

\section{ABSTRACT}

\section{ANALYSIS OF EMBRYO PRODUCTION IN VITRO FERTILIZATION AND EMBRYO TRANSFER TO NELLORE DONORS}

A probability density function for the number of viable embryos produced after an in vitro fertilization program in Nellore donors was adjusted through data provided by the Brazilian Association of Zebu breeders. Results were based on 20,619 donors, 71,602 aspirations and the total of 509,643 embryos. The probability density function of the number of viable embryos was modeled using exponential distribution. Parameters fitting were carried out for the maximum likelihood using a non-linear gradient method. The precision level was $\mathrm{RMSE}=0.040$ and $\mathrm{R}^{2}=0.98$

KEY WORDS: Data bank, donors, probability density, simulation.

\section{INTRODUÇÃO}

Na bovinocultura nacional, diversos avanços têm sido demonstrados na fertilização in vitro (FIV), for the representation of probability of number of viable embryos produced by Nellore donors by in vitro fertilization technique. To analyze probability density of embryo recovery (Beltrame, 2006) with in vitro fertilization adjust was used the curve comparison test through F test (Silva and Azevedo, 2002). There were no differences between the curves. These results suggest that there may be a unique and restricting factor that affects biologically the embryo production on the embryo transfer and in vitro fertilization techniques. transferência de embriões (TE) e sexagem de espermatozoide. Entretanto, a incerteza dos resultados é permanente, o que gera imprecisões nas respostas simuladas, quando comparadas aos resultados observados. Essas 
distinções têm sido minimizadas quando metodologias corretas de simulação são implementadas (BELTRAME, 2006; BELTRAME, 2007; BARIONI, 2007).

Durante o processo de FIV de embriões de uma doadora qualquer, é impossível prever com exatidão o número de oócitos que serão aspirados nem o número de embriões viáveis a serem produzidos. Inúmeras decisões pertinentes à FIV e à TE são dependentes dessa informação (número de receptoras a sincronizar, custo, número de embriões a congelar, material utilizado, tempo etc.). Mesmo assim, em algumas biotécnicas da reprodução animal, é necessário fazer previsões a respeito do valor futuro de certas variáveis, tomando por base dados históricos (BELTRAME et al., 2007). Fica evidente, portanto, a necessidade de um estudo mais apurado do comportamento de uma série de variáveis aleatórias, objetivando determinar seu comportamento em cenários simulados e não sua utilização como variáveis deterministas.

Uma vez definida uma variável aleatória, existe interesse no cálculo dos valores das probabilidades associadas a essa variável. O conjunto das variáveis e das probabilidades correspondentes é denominado distribuição de probabilidades, isto é: $\left\{\left(\mathrm{x}_{\mathrm{i}} \mathrm{p}\left(\mathrm{x}_{\mathrm{i}}\right), \mathrm{I}=1,2, \ldots \mathrm{n}\right\}\right.$. Neste caso, a função de densidade de probabilidade é a função que associa a cada valor assumido pela variável aleatória a probabilidade do evento correspondente, tal como: $\mathrm{P}\left(\mathrm{X}=\mathrm{x}_{\mathrm{i}}\right)=\mathrm{P}\left(\mathrm{A}_{\mathrm{i}}\right), \mathrm{i}=1,2, \ldots, \mathrm{n}($ FREITAS FILHO, 2001; LEVINE et al., 2005).

A simulação de sistemas requer o uso de sequências de valores de determinadas variáveis aleatórias. Podem ser considerados três modos de obtê-las: o uso de sequências provenientes de observações efetuadas previamente; sequências geradas aleatoriamente a partir de distribuições empíricas construídas com observações efetuadas previamente; sequências geradas aleatoriamente a partir de distribuições clássicas, cujos parâmetros foram estimados de acordo com observações efetuadas previamente (PERIN FILHO, 1995).

No primeiro caso, exige-se um processo demorado, que requer bastante memória e que, portanto, é inadequado para a aplicação em simulação de sistemas. As outras duas alternativas são dependentes de um gerador de números aleatórios, que permite obter valores de variáveis aleatórias com as distribuições desejadas.

A determinação da curva de densidade pro- babilidade da variável número de embriões viáveis objetiva sua projeção de forma realista em estudos de simulação. Assim, estimativas do número de prenhezes, número de receptoras e doadoras a se utilizar, custo da técnica, elaboração de projetos e cálculos de viabilidade financeira podem ser realizados com maior precisão, minimizando risco (BELTRAME 2006).

Neste contexto, o objetivo deste trabalho é determinar a curva de densidade probabilidade do número de embriões viáveis produzidos por FIV para doadoras da raça Nelore, efetuando sua comparação com a curva de densidade probabilidade da TE, determinada por BELTRAME (2006).

\section{MATERIAIS E MÉTODOS}

O presente trabalho toma por base dados concedidos pela Associação Brasileira de Criadores de Zebu $(\mathrm{ABCZ})$ relativo à FIV em doadoras da raça Nelore. Ainda, a frequência do número de embriões viáveis obtidos após TE e descrita por BELTRAME (2006) foi utilizada neste trabalho.

Por intermédio da $\mathrm{ABCZ}$, uma base de dados compondo arquivos eletrônicos em formato " $t x t$ ", com dados de embriões FIV, foram recebidos contendo, na linha, o evento, e nas colunas a seguinte padronização: código da doadora; código do touro; uf da fazenda; data da aspiração; data da transferência; embriões produzidos; embriões transferidos; embriões perdidos.

Organizaram-se a informações relativas à FIV em planilhas do Microsoft Excel 2003, e a partir desse ponto, procedeu-se a sua análise por meio da ferramenta SAS for Windows 1999. Dados de 73.121 aspirações foram disponibilizados.

Uma vez na ferramenta SAS, eliminaram-se da análise todos os dados incoerentes ou de valor nulo. Consideraram-se apenas as aspirações realizadas entre os anos de 2000 a 2007.

Foram retiradas da análise as aspirações em que o número de embriões obtidos e/ou perdidos era superior a 35, apresentavam datas inconsistentes e/ ou valores em branco.

A aplicação da restrição resultou na eliminação de 1.519 aspirações. A análise partiu, então, de dados referentes a 20.619 doadoras, 71.602 aspirações e 509.643 embriões.

A seleção dos dados relevantes foi realizada através 
da função "proc freq" do SAS for Windows 1999. No caso específico, essa função retornou o somatório do número observado de aspirações para cada valor de número de embriões viáveis gerados. O número total de aspirações que obtiveram embrião viável nulo, até o somatório de aspirações que obtiveram 35 embriões viáveis, número máximo de embriões viáveis por doadora restringido no banco, foi gerado a partir desse "comando".

Uma comparação gráfica e uma análise numérica baseada na frequência observada e estimada a partir do modelo de distribuição (ou distribuições) selecionada foram realizadas. Definiu-se por estimar a frequência nula como sendo similar à frequência de obtenção de um embrião viável e a partir daí realizar seu ajuste.

O comportamento gráfico dos dados observados torna-se fundamental para um diagnóstico preliminar da escolha de uma distribuição, conforme é demonstrado na Figura 1.

A disposição gráfica das diversas distribuições existentes e o comportamento dos dados observados sugerem uma aproximação à distribuição exponencial negativa [ $E X(\lambda)$ ] (BENJAMIN \& CORNELL, 1970). Sendo assim, uma metodologia numérica foi utilizada, no intuito de identificar o melhor valor dos parâmetros e comparar o ajuste desses modelos aos dados observados.

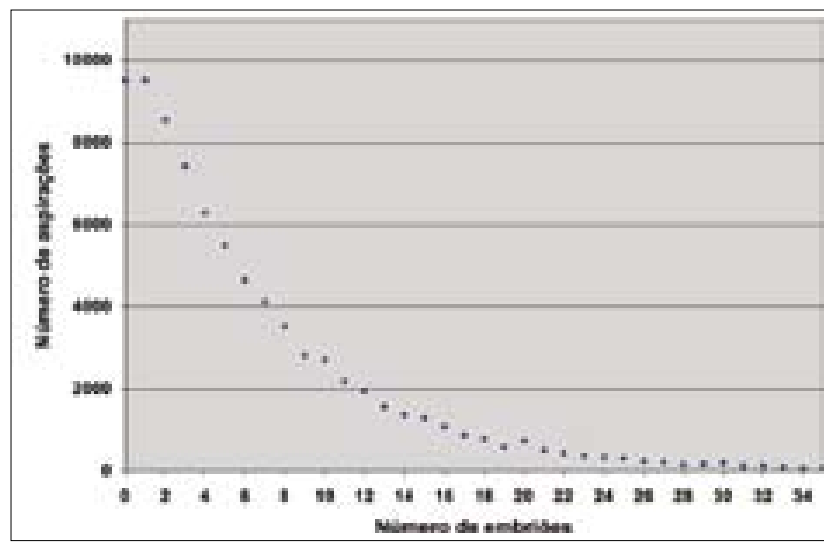

FIGURA 1. Frequência observada da produção embrionária em relação ao número de aspirações realizadas para técnica de fertilização in vitro.

Procedeu-se à estimação dos parâmetros da distribuição e seu ajuste, para representar com eficiência a variância e minimizar o erro total, através do método da "máxima verossimilhança" e de seu estimador (KOSTINA, 2004). O método GRG2 como instrumento de otimização foi aplicado usando-se Microsoft Excel $2003^{\circledR}$ Solver.

Os procedimentos de ajuste descritos foram resumidos e podem ser observados na Tabela 1, para o uso de uma distribuição exponencial [ $E X(\lambda)]$.

TABELA 1. Procedimentos de ajuste da distribuição exponencial negativa do número de embriões viáveis na técnica de fertilização in vitro

\begin{tabular}{|c|c|c|c|c|}
\hline $\mathrm{N}^{\mathrm{o}}$. de embriões (1) & $\begin{array}{c}\text { Frequência } \\
\text { observada (2) }\end{array}$ & $\begin{array}{l}\text { Frequência estimada } \\
\text { (3) }\end{array}$ & Erro de predição (4) & $\begin{array}{l}\text { Erro } \\
(5)\end{array}$ \\
\hline 0 & 0,118 & $F(x)=1-e^{-1 x}, x>0$ & (3)-(2) & (4) \\
\hline 1 & 0,118 & $F(x)=1-e^{-1 x}, x>0$ & (3)-(2) & (4) \\
\hline 2 & 0,105 & $F(x)=1-e^{-1 x}, x>0$ & (3)-(2) & (4) \\
\hline$\ldots$ & $\ldots$ & $\ldots$ & & Serro \\
\hline
\end{tabular}

(1) $\mathrm{N}^{\mathrm{o}}$. de embriões viáveis por aspiração; (2) frequência observada dos dados coletados; (3) frequência estimada a partir da distribuição de probabilidade; (4) erro de predição; (5) erro total.

Estatisticamente, após a parametrização do modelo, procedeu-se ao teste $\mathrm{F}$ e calculou-se o coeficiente de determinação. Assim, foram determinados o quanto da variância obtida na parametrização era explicada pelo modelo e a significância dos dados obtidos pela nova distribuição.

Como critérios de escolha da melhor função de distribuição que se ajusta aos dados observados, foram avaliados, em fator de importância, respectivamente, a soma do quadrado do erro (SQerro) e o erro de predição.

$\Sigma \mathrm{EP}=\frac{\text { frequência observada }- \text { frequência estimada }}{\text { frequência observada }} \times 100$ 
Comparação dos modelos

BELTRAME (2006) descreveu um banco de dados de TE ajustado por meio de um aplicativo de gerenciamento de banco de dados. Neste trabalho, optou-se por um software livre, modelado por meio da ferramenta IBAdmin 4.0, sendo analisadas 26.767 doadoras, 61.928 coletas e 451.322 embriões.

Conforme BELTRAME (2006), o ajuste da TE foi realizado através da distribuição exponencial negativa. Assim, de posse de um mesmo modelo e de dois (ou mais) conjuntos de dados (TE e FIV), objetivou-se saber se estes conjuntos de dados se comportariam da mesma forma.

Assim, para comparar a significância estatística das diferenças entre os modelos (curvas de probabilidade de TE ajustada por BELTRAME, em 2006, e de FIV, neste trabalho), aplicou-se uma nova análise estatística, qual seja, a técnica de comparação de curvas com o teste F. Representou-se o teste de verossimilhança através da seguinte equação (SILVA \& AZEVEDO, 2002):

$$
\mathrm{F}=\frac{\left[\mathrm{SQR}_{(\text {comb })}-\left(\mathrm{SQR}_{E}+\mathrm{SQR}_{F I V}\right] / 1\right.}{\left(\mathrm{SQR}_{E}+\mathrm{SQR}_{F I V}\right) / 35},
$$

em que $F$ é o valor da distribuição $\mathrm{F}$ correspondente à razão da diferença entre a soma dos quadrados residuais da curva combinada; $S Q R_{E}$ é a soma dos quadrados residuais da curva de probabilidade de TE; $S Q R_{F I V}$ é a soma dos quadrados residuais da curva de probabilidade de FIV; $S Q R_{(c o m b)}$ é a soma dos quadrados residuais da curva de probabilidade resultante do ajuste com os dados proveniente da curva de TE e FIV combinadas. Os divisores 1 e 35 representam os respectivos graus de liberdade do numerador e denominador, utilizados no cálculo da razão entre os quadrados médios residuais de cada par de curvas comparadas. O nível crítico de probabilidade estabelecido para julgar a significância das diferenças foi de $5 \%$, o qual, nesse caso, correspondeu ao valor de $\mathrm{F}=4,13$ para 1 e 34 graus de liberdade no numerador e denominador, respectivamente.

\section{RESULTADOS E DISCUSSÃO}

A frequência observada corresponde à demonstração do evento específico no passado. Os dados obti- dos representam o somatório do número de coletas com embriões viáveis, variando de 0 a 35 . Tais informações possibilitaram a determinação da curva de densidade probabilidade. A Tabela 2 apresenta estes dados.

TABELA 2. Frequência observada do número de embriões viáveis por aspiração para a raça Nelore

\begin{tabular}{|c|c|c|}
\hline $\begin{array}{l}\text { Embriões } \\
\text { viáveis }\end{array}$ & Total de aspirações & $\begin{array}{c}\text { Frequência } \\
\text { observada (\%) }\end{array}$ \\
\hline 0 & 448 & 0,63 \\
\hline 1 & 9.531 & 13,31 \\
\hline 2 & 8.542 & 11,93 \\
\hline 3 & 7.431 & 10,38 \\
\hline 4 & 6.300 & 8,8 \\
\hline 5 & 5.494 & 7,67 \\
\hline 6 & 4.660 & 6,51 \\
\hline 7 & 4.125 & 5,76 \\
\hline 8 & 3.548 & 4,96 \\
\hline 9 & 2.820 & 3,94 \\
\hline 10 & 2.704 & 3,78 \\
\hline 11 & 2.194 & 3,06 \\
\hline 12 & 1.955 & 2,73 \\
\hline 13 & 1.573 & 2,2 \\
\hline 14 & 1.376 & 1,92 \\
\hline 15 & 1.292 & 1,8 \\
\hline 16 & 1.098 & 1,53 \\
\hline 17 & 878 & 1,23 \\
\hline 18 & 789 & 1,1 \\
\hline 19 & 589 & 0,82 \\
\hline 20 & 742 & 1,04 \\
\hline 21 & 507 & 0,71 \\
\hline 22 & 438 & 0,61 \\
\hline 23 & 369 & 0,52 \\
\hline 24 & 338 & 0,47 \\
\hline 25 & 314 & 0,44 \\
\hline 26 & 241 & 0,34 \\
\hline 27 & 220 & 0,31 \\
\hline 28 & 161 & 0,22 \\
\hline 29 & 176 & 0,25 \\
\hline 30 & 200 & 0,28 \\
\hline 31 & 124 & 0,17 \\
\hline 32 & 136 & 0,19 \\
\hline 33 & 108 & 0,15 \\
\hline 34 & 81 & 0,11 \\
\hline 35 & 100 & 0,14 \\
\hline
\end{tabular}

Como observado na Tabela 2, a frequência de embriões nulos apresenta-se como um dado atípico na tabela $(\mathrm{f}(0)=0,63)$. Embora passível de ocorrência, sugere-se que tal dado seja proveniente da falha de comunicação por veterinários e fazendeiros das aspirações que apresentam número de embriões viáveis 
nulos, apresentando, portanto, frequência extremamente baixa.

No processo de análise dos dados coletados para uso em um simulador em análises estocásticas, uma das principais ações é a identificação de uma distribuição teórica de probabilidades que represente, da melhor maneira possível, o comportamento da variável (FREITAS FILHO, 2001).

Para FIV, a média de embriões viáveis por aspiração revelados pela frequência observada foi de 6,43 $\pm 5,5$. A obtenção dessa média teve como pressuposto que o número de aspirações que resultaram em zero e um embriões foi similar. O parâmetro de ajuste do modelo para FIV foi $\mathrm{k}=0,148$ para a distribuição exponencial negativa. Considerou-se o ajuste altamente significativo ( $\mathrm{P}<0,0001$; Tabela 3 e Figura 2$)$.

O coeficiente de determinação também pode ser considerado alto $\left(\mathrm{R}^{2}=0,98\right)$, indicando que a distribuição explica a maior parte da variância nos dados observados. O erro-padrão da regressão foi de 0,040 .

Tenta-se predizer a eficiência dos programas de FIV por medida em relação ao número de blastocistos obtidos a partir do número de ovócitos postos em cultivo e fertilizados com um sêmen determinado (FUENTES, 2006). Entretanto, o comportamento dessas variáveis não é conhecido na técnica de FIV.

A média de embriões viáveis obtidos por aspiração neste trabalho encontra-se próxima à relatada na literatura. NONATO JR. et al. (2006) compararam quatro protocolos de OPU em vacas Nelore. O número de embriões produzidos variou de $6,1 \pm 1,0$ a $12,0 \pm$ 2,6, este último com utilização de progestágeno.

O grupo de MACHADO et al. (2006), intencionando estudar a variabilidade na produção in vitro, analisou a hipótese de que uma menor variabilidade ocorreria entre gêmeos idênticos do que quando indivíduos não relacionados eram trabalhados. Diferença $(\mathrm{P}<0,05)$ foi encontrada no número de blastocistos produzidos entre os grupos comparados.

CHAUBAL et al. (2007) compararam tratamentos hormonais na tentativa de promover estimulação ovárica e aumentar a produção de embriões na técnica de FIV. Embora um número menor de embriões tenha sido produzido diante dos tratamentos utilizados, a variabilidade na produção de embriões persistiu.

Fica clara a importância do conhecimento do comportamento da variável e suas projeções dentro de sistemas de simulação. Através da Figura 2, demonstrase que existe um comportamento característico da variável analisada independente do sistema em estudo. Este comportamento é decrescente e proporcional ao aumento no número de embriões produzidos, sendo ajustável a situações específicas. Ainda, os resultados deste trabalho indicam que o número de embriões viáveis produzidos por aspiração de doadoras da raça Nelore pode ser estimado por meio da distribuição exponencial.

TABELA 3. Caracteres da análise de variância para a distribuição exponencial ajustada do número de embriões viáveis por aspiração

\begin{tabular}{lccccc}
\hline \multicolumn{1}{c}{ FV } & GL & SQ & QM & F & P $<$ \\
\hline Regressão & 1 & 0,04023 & 0,0402 & 2510,9 & $0.0001^{* *}$ \\
Erro & 34 & 0,00062 & 0,000016 & - & - \\
Total & 35 & 0,04085 & - & - & - \\
R $^{2}$ & 0,98471 & & - & - & -
\end{tabular}

$\mathrm{FV}=$ fonte de variação; $\mathrm{GL}=$ grau de liberdade; $\mathrm{SQ}=$ soma do quadrado do erro; $\mathrm{QM}=$ quadrado médio; $\mathrm{F}=$ teste de significância; $* *$ = probabilidade $\mathrm{P}<0,0001$.

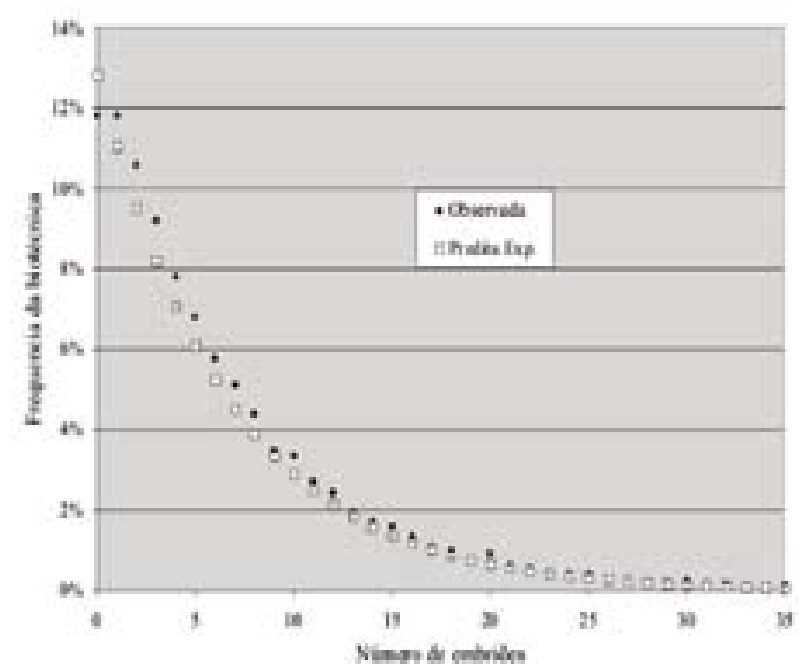

FIGURA 2. Comparação entre a frequência observada e a frequência estimada do número de embriões viáveis por aspiração obtida pela distribuição exponencial.

Comparação de modelos

A Figura 3 e os resultados obtidos decorrentes da avaliação de comparação dos modelos indicam 
a não significância da hipótese testada, ou seja, não existe diferença $(\mathrm{P}>0,05)$ entre as curvas de TE e FIV analisadas. A demonstração estatística do resultado pode ser observada na Tabela 4 .

TABELA 4. Caracteres da análise de variância para modelos combinados do número de embriões viáveis produzidos após coleta e aspiração

\begin{tabular}{lccccc}
\hline \multicolumn{1}{c}{ FV } & GL & SQ & QM & F & P> \\
\hline Regressão & 1 & 0,0853 & 0,0853 & 0,81 & $0,05^{\text {ns }}$ \\
Erro & 34 & 0,0024 & 0,0000715 & - & - \\
Total & 35 & 0,0877 & - & - & - \\
$\mathrm{R}^{2}$ & 0,9723 & & - & - & - \\
\hline
\end{tabular}

$\mathrm{FV}=$ fonte de variação; $\mathrm{GL}=$ grau de liberdade; $\mathrm{SQ}=$ soma do quadrado do erro; $\mathrm{QM}=$ quadrado médio; $\mathrm{F}=$ teste de significância; $\mathrm{ns}=$ probabilidade $\mathrm{P}>0,05$ - não significativo

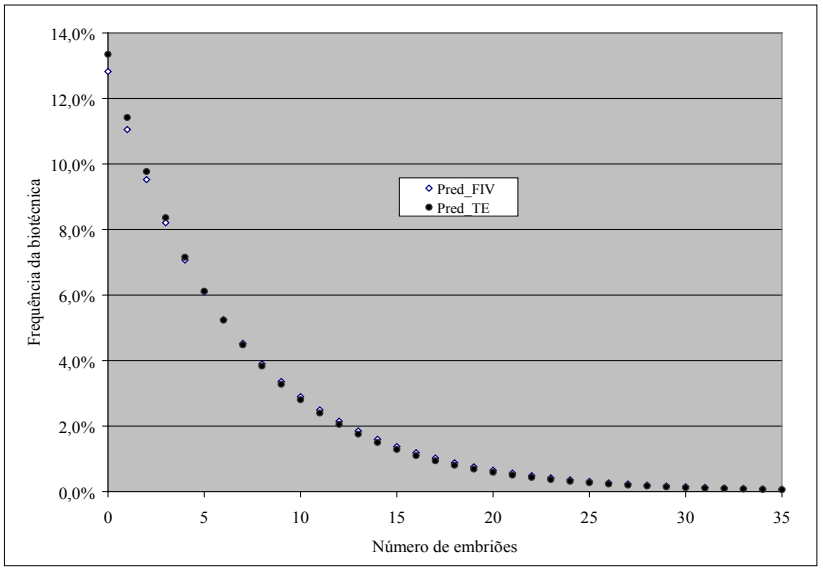

FIGURA 3. Frequência predita através da distribuição exponencial do número de embriões viáveis produzidos após transferência de embriões e fertilização in vitro.

É importante destacar que estudo como este é incipiente na esfera da reprodução animal. Embora matematicamente se chegue a um resultado pontual, a explicação biológica de similaridade de curvas engloba uma série de suposições.

Pelos resultados apresentados, inicialmente, supõe-se que exista um processo em comum que limite o número de embriões viáveis nas duas técnicas. Esta ideia pode ser consequência de fatores que influenciam a dinâmica folicular na resposta ovulatória de doadoras, fatores que afetam a fertilização e a viabilidade embrionária, e ainda fatores relacionados ao programa de manejo animal, como descrevem PEIXOTO et al. (2002).

Tendo-se a dinâmica folicular como fator limitante na produção de embriões, a elaboração de modelos matemáticos que retratassem a evolução do crescimento folicular de forma individual, excluindo-se o comportamento da população, poderia ser útil para estimar de forma mais precisa o número de embriões viáveis obtidos por coleta. Entretanto, estudos visando tal modelagem são complexos e ainda não foram descritos na medicina veterinária.

Em relação à similaridade das curvas, um dos pontos que se cogita é que, diante da projeção do número de embriões viáveis na TE ou na FIV, o número de embriões gerados através de processo aleatório possuirá o mesma capacidade de evolução. Neste caso, a dinâmica temporal deve ser alterada. A aceleração do melhoramento genético animal fica evidenciada pelo tempo (repetição) e não pela técnica utilizada. Em situações similares em que os efeitos genético e ambiental não forem considerados, a produção embrionária projetada em um modelo de simulação pode ser a mesma.

Ainda quanto à comparação FIV e TE, os resultados permitem identificar as biotécnicas como auxiliares e não como conflitantes. Neste caso, ineficiências de uma biotécnica podem ser cobertas pelos benefícios da outra. Animais que não respondem à $\mathrm{TE}$ podem ser utilizados na FIV. Da mesma forma, animais com consecutivas TEs podem ser inseminados e ao início da gestação serem submetidos à FIV. Com isso, uma maior velocidade de seleção pode ser obtida. Destaca-se que outros bancos de dados devem ser testados no intuito de confirmar os resultados obtidos neste estudo.

Ressalta-se que uma maior atenção deve ser destinada aos índices das biotécnicas. Embora não tenham sido demonstradas diferenças nas curvas de produção de embriões entre TE e FIV, algumas variáveis como taxas de gestação, utilização de doadoras consecutivamente e possibilidade de congelamento de embriões podem ser decisivas na escolha por uma das biotécnicas de forma específica.

\section{CONCLUSÕES}

Os resultados expostos permitem concluir que: 1) a distribuição de densidade de probabilidade expo- 
nencial se mostra adequada ao ajuste à frequência observada para a finalidade proposta (uso em simulação) na biotécnica de FIV; 2) existe grande proximidade de comportamento do número de embriões viáveis obtidos após coleta e aspiração entre as curvas de TE e FIV testadas.

\section{AGRADECIMENTOS}

À Associação Brasileira dos Criadores de Zebu (Sr. Carlos Henrique Cavalari), pela concessão e permissão de uso dos dados. Ao CNPq, pela concessão da bolsa de estudos do primeiro autor.

\section{REFERÊNCIAS}

BARIONI, L.G.; BELTRAME, R.T.; QUIRINO, C.R.; FERNANDES, D.R. Modelos determinista e estocástico em programas de transferência de embriões em bovinos. Archivos Latinoamericanos de Producción Animal, v. 15, p. 107-113, 2007.

BELTRAME, R. T. Simulação bioeconômica da transferência de embriões em bovinos da raça Nelore. 2006. 86 f. Dissertação (Mestrado)-Universidade Estadual do Norte Fluminense (UENF), Campos dos Goytacazes, 2006.

BELTRAME, R.T.; BARIONI, L.G.; MAESTRI, B.D.; QUIRINO, C.R. Economic optimization of the number of recipientes in bovine embryo transfer programs. Scientia Agricola, v. 64, p. 221-226, 2007.

Benjamin, J. R.; CORNELL, C. A. Probability, statistics and decision for engineers. San Francisco, USA: McGraw-Hill Book, 1970.
CHAUBAL, S. A.; FERRE, L. B.; MOLINA, J. A.; FABER, D. C.; BOLS, P. E. J.; REZAMAND, P.; TIAN, X.; YANG, X. Hormonal treatments for increasing the oocyte and embryo production in an OPU-IVP system, Theriogenology, v. 67, p. 719-728, 2007.

DE LA FUENTE, J. Reproduccion asistida en bovinos. In: CURSO INTERNACIONAL DE REPRODUCCIÓN ANIMAL, 30., 2007, Madrid. Anais..., Madrid: INIA, 2007.

FREITAS FILHO, P.J. Introdução à modelagem e simulação de sistemas (com aplicações em Arena). Santa Catarina: Visual Books, 2001.

KOSTINA, E. Robust parameter estimation in dynamic systems. Optimization and Engineering, v. 5, p. 461-484, 2004.

LEVINE, D.; M.; STEPHAN, D.; KREHBIEL, T. C.; BERENSON, M. L. Estatística: teoria e aplicações usando o Microsoft Excel em português. Rio de Janeiro: LTC, 2005.

MACHADO, S.A.; REICHENBACH, H.D.; WEPPERT, M.; WOLF, E.; GONÇALVES, P.B.D. The variability of ovum pick-up response and in vitro embryo production from monozygotic twin cows. Theriogenology, v. 65, p. 573-583, 2006.

PEIXOTO, M.G.C.D.; FONSECA, C.G.; PENNA, V.M.; ALVIM, M.T.T. Análise multivariada de resultados da ovulação múltipla seguida de transferência de embriões de doadoras zebuínas. Arquivo Brasileiro de Medicina Veterinária e Zootecnia, v. 54, n. 5, 2002.

PERIN FILHO, C. Introdução à simulação de sistemas. Campinas, SP: Editora da Unicamp, 1995. 163 p.

NONATO JR. I.; PONTES, J. H. F.; ERENO JR., J. C.; GIMENES, L. U. G.; TORRES JR., J. R. S.; BARUSELLI, P. S. Utilização de progesterona exógena em protocolos de OPU de vacas Nelore: resultados preliminares. Acta Scientiae Veterinariae, v. 34, Supl. 1, p. 452, 2006. 